\title{
Radiomic assessment as a method for predicting tumor mutation burden (TMB) of bladder cancer patients: a feasibility study
}

\author{
Xin Tang ${ }^{1}$, Wen-lei Qian', Wei-feng Yan ${ }^{1}$, Tong Pang ${ }^{1}$, You-ling Gong ${ }^{2^{*+}}$ and Zhi-gang Yang ${ }^{1{ }^{*+}}$
}

\begin{abstract}
Background: Tumor mutation burden (TMB) is an emerging prognostic biomarker of immunotherapy for bladder cancer (BLCA). We aim at investigating radiomic features' value in predicting the TMB status of BLCA patients.

Methods: Totally, 75 patients with BLCA were enrolled. Radiomic features extracted from the volume of interest of preoperative pelvic contrast-enhanced computed tomography (CECT) were obtained for each case. Unsupervised hierarchical clustering analysis was performed based on radiomic features. Sequential univariate Logistic regression, the least absolute shrinkage and selection operator (LASSO) regression and the backward stepwise regression were used to develop a TMB-predicting model using radiomic features.

Results: The unsupervised clustering analysis divided the total cohort into two groups, i.e., group A (32.0\%) and B (68.0\%). Patients in group A had a significantly larger proportion of having high TMB against those in group $B$ (66.7\% vs. $41.2 \%, p=0.039)$, indicating the intrinsic ability of radiomic features in TMB-predicting. In univariate analysis, 27 radiomic features could predict TMB. Based on six radiomic features selected by logistic and LASSO regression, a TMB-predicting model was built and visualized by nomogram. The area under the ROC curve of the model reached 0.853 . Besides, the calibration curve and the decision curve also revealed the good performance of the model.

Conclusions: Our work firstly proved the feasibility of using radiomics to predict TMB for patients with BLCA. The predictive model based on radiomic features from pelvic CECT has a promising ability to predict TMB. Future study with a larger cohort is needed to verify our findings.
\end{abstract}

Keywords: Bladder cancer, Radiomics, Tumor mutation burden, Driver mutations, Predictive model

\section{Background}

The past several years have witnessed the blooming of immune checkpoint inhibitors (ICIs) targeting the programmed death-1 (PD-1) pathway in cancer treatment [1]. As the most common tumor of the urinary system,

\footnotetext{
*Correspondence: gongyouling@hotmail.com; yangzg666@163.com

${ }^{+}$You-ling Gong and Zhi-gang Yang contributed equally to this work.

${ }^{2}$ Department of Thoracic Oncology and State Key Laboratory of Biotherapy, Cancer Center, West China Hospital, Sichuan University, 37\# Guo Xue Xiang, Chengdu 610041, Sichuan, China

'Department of Radiology, West China Hospital, Sichuan University, 37\# Guo Xue Xiang, Chengdu 610041, Sichuan, China
}

bladder cancer (BLCA) is recognized as one of the immunologically "hot" tumors [2], and thereby, a good candidate for immunotherapy. Till now, the FDA has approved three programmed death-L1 (PD-L1) inhibitors, i.e. atezolizumab, durvalumab, and avelumab, as well as two PD-1 inhibitors i.e. nivolumab and pembrolizumab in the treatment of urothelial carcinoma [3].

Although the emergence of immunotherapy brings hope for patients of BLCA, it cannot be ignored that this novel treatment is not always effective in all patients. Besides, immunotherapy can also cause toxic and

C C The Author(s). 2021 Open Access This article is licensed under a Creative Commons Attribution 4.0 International License, which permits use, sharing, adaptation, distribution and reproduction in any medium or format, as long as you give appropriate credit to the original author(s) and the source, provide a link to the Creative Commons licence, and indicate if changes were made. The images or other third party material in this article are included in the article's Creative Commons licence, unless indicated otherwise in a credit line to the material. If material is not included in the article's Creative Commons licence and your intended use is not permitted by statutory regulation or exceeds the permitted use, you will need to obtain permission directly from the copyright holder. To view a copy of this licence, visit http://creativecommons.org/licenses/by/4.0/ The Creative Commons Public Domain Dedication waiver (http://creativecommons.org/publicdomain/zero/1.0/) applies to the data made available in this article, unless otherwise stated in a credit line to the data. 
potentially fatal side effects [4]. Therefore, identifying biomarkers that can distinguish the potential responders of ICIs from the non-responders is essential for accurate treatment decisions. PD-L1 expression and tumor mutation burden (TMB) are the two most commonly used biomarkers $[5,6]$. Unlike the detection of PD-L1 expression that focuses on the targeting protein of the ICIs, TMB predicts the therapeutic efficacy of ICIs through its strong correlation to the mutation-derived neoantigens which is a key factor for immune response activation [5]. Studies even showed that TMB is superior to PD-L1 in predicting the therapeutic efficacy of ICIs therapy $[7,8]$. Besides, it has been reported that higher TMB is associated with a favorable prognosis of ICIs treatment in tumors including melanoma, non-small-cell lung cancer, small-cell lung cancer, urothelial cancer [9-13] and etc. However, a major obstacle that prevents the large-scale promotion of TMB detection in patients receiving ICIs is the high cost of the whole-exome sequencing (WES) test.

Radiomics is a rapidly emerging field that can be applied to many biomedical areas [14]. Studies have shown that radiomic features are capable of predicting the somatic mutation of certain genes in different tumor types [15-17]. Moreover, two latest studies addressed that radiomics could also predict the TMB status of lung cancer patients $[18,19]$. These studies revealed the deep connection between the radiomic and genomic characteristics in cancer patients and the feasibility of using radiomic features to predict the genomic outcomes. Therefore, we hypotheses that radiomic features can be used to select the most clinically needed patient population for TMB testing by predicting the probability of high TMB. Besides, radiomics may act as an alternative or assistant diagnosis of TMB detection for those who are not accessible to the expensive WES test.

In this study, we aim at exploring the value of radiomic features extracted from pelvic contrast-enhanced computed tomography (CECT) images in predicting the TMB status of BLCA patients, and also, developing a TMB-predicting model based on the radiomic data.

\section{Methods}

\section{Study population and data acquisition}

Pelvic CECT images of eligible BLCA patients were downloaded from the Cancer Imaging Archive database (TCIA, http://www.cancerimagingarchive.net/) [20]. Genetic and clinical data were acquired from the Cancer Genome Atlas (TCGA) database (http://cancergenome. nih.gov) [21]. The Inclusion criteria are shown in Fig. 1A, including: 1) pathological diagnosis as BLCA; 2) available preoperative pelvic CECT images with good quality; 3 ) available genetic information. Finally, 75 eligible BLCA patients from the TCGA-BLCA cohort were enrolled. No ethical approval nor informed consent was required for the current study due to the public availability of data in the TCIA and TCGA databases.

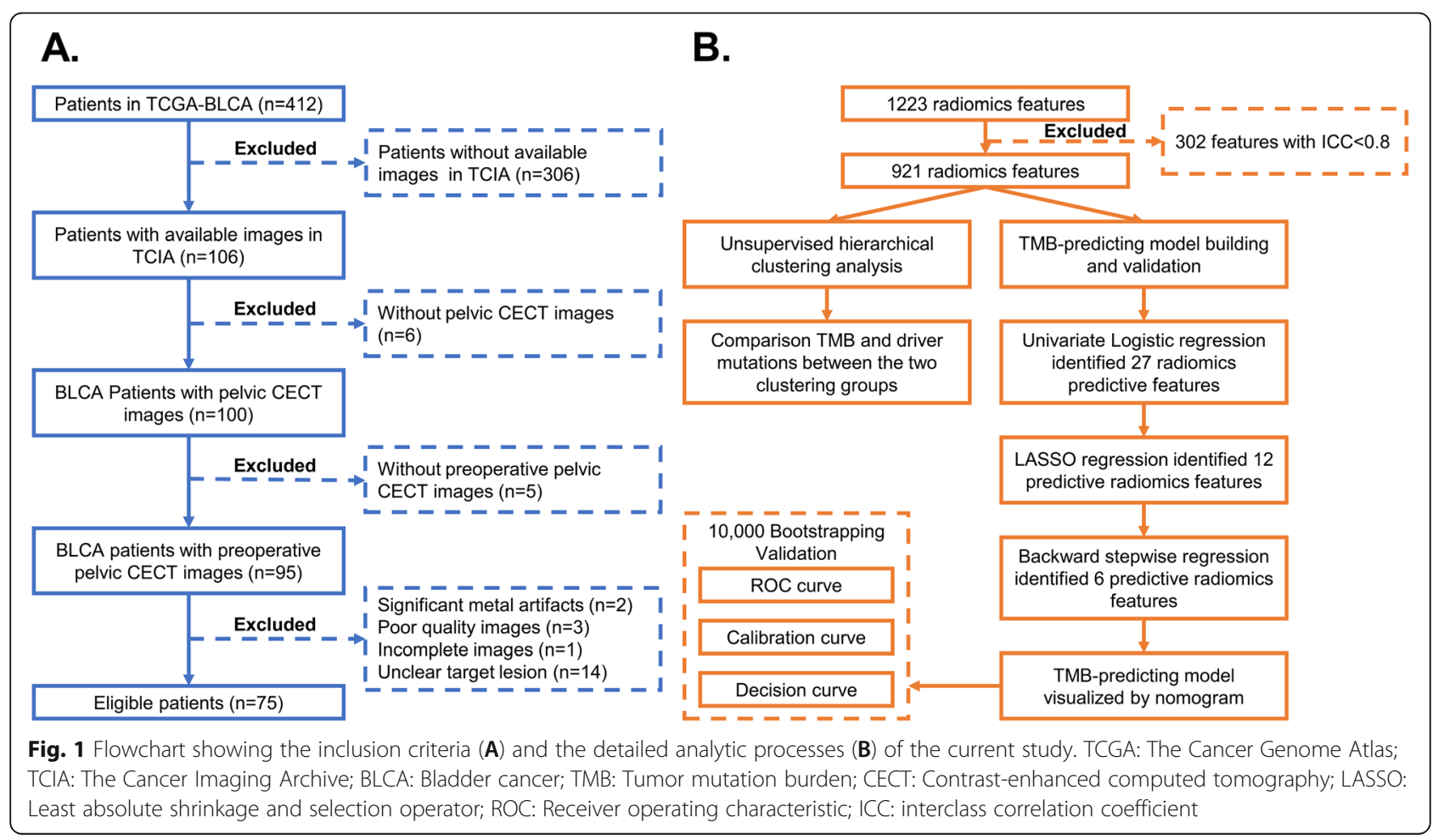




\section{CT imaging parameters}

The preoperative pelvic CECT images were obtained from four various manufactures: General Electric (GE), Siemens, Philips and Toshiba Medical Systems. The acquisition parameters of CT were as follows: slice thickness, 1.25 - $5 \mathrm{~mm}$; tube voltage, $100-140 \mathrm{kV}$; tube current, 80-689 mA; matrix, $512 \times 512$; and pixel size, $0.586 \times 0.586 \mathrm{~mm}^{2}$ to $0.977 \times 0.977 \mathrm{~mm}^{2}$.

\section{The volume of interest (VOI) delineation and feature extraction}

All pelvic CECT images were obtained before surgery. On the axial CECT image, two radiologists used the open-source software 3D slicer (Version 4.10.2) [22] to manually delineate the VOI of tumor independently.

Imaging pre-processing steps were conducted to decrease the potential protocol variability. Prior to feature extraction, all CT images were resampled into voxel sizes $1 \times 1 \times 1 \mathrm{~mm}^{3}$ and discretized to a bin width of 25 Hounsfield units (HU). Finally, 1223 radiomic features including shape, first order and texture features with and without performing Wavelet and Laplacian of Gaussian (LoG) filter were acquired. Texture features were classified into five categories including gray-level co-occurrence matrix (GLCM), gray-level difference matrix (GLDM), gray-level run length matrix (GLRLM), gray-level size zone (GLSZM) and neighborhood gray tone difference Matrix (NGTDM).

Combat algorithm was applied for feature harmonization in different imaging protocols from multicenter investigations (https://github.com/Jfortin1/ ComBatHarmonization) [23, 24]. All the radiomic features were normalized by the Z-score transformation. The consistency of radiomic features between the two radiologists was assessed by interclass correlation coefficient (ICC). Only stable features with ICC $>0.8$ were included in the further analysis (Fig. 1B).

\section{Tumor mutation burden}

In this study, TMB calculation was based on somatic nonsynonymous mutation, while synonymous mutation was excluded. For each patient, TMB was counted as the total mutational count divided by the exome size (estimated as $38 \mathrm{Mb}$ ) [25]. Based on the median TMB of all patients, the total cohort was divided into high and low TMB groups. Besides, the top 20 driver mutations of BLCA (obtained from the driver mutation database IntOGen [26]) were also included in the analyses.

\section{Unsupervised hierarchical clustering analysis}

Unsupervised hierarchical clustering analysis was performed to identify distinct subgroups of BLCA patients based on the homogeneity and heterogeneity of the radiomic features. Genomic outcomes and clinical data were compared between the clustering groups by chisquare test. Based on the similarity of radiomics among samples calculated by Euclidean distance, hierarchical clustering can split the total cohort into different subgroups with high radiomic similarity within each subgroup while distinct radiomic profile between subgroups. Unsupervised hierarchical clustering analysis was conducted using the "pheatmap" R package.

Development and validation of the TMB-predicting model The detailed produces of radiomic TMB-predicting model building were described as follow: Firstly, univariate logistic regression analysis was used to preliminarily screen and identify potential TMB-predictors from radiomic features. Then radiomic features with $p<0.05$ in univariate analysis were further examined by the least absolute shrinkage and selection operator (LASSO) regression methods via 10-fold cross-validation based on minimum criteria. In addition, multivariate logistic regression using backward elimination strategy was performed to eliminate the redundant features. Finally, TMB-predicting model based on simplified radiomic features was established.

The novel TMB-predicting radiomic model was visualized as nomogram. Besides, the performance of this predictive model was evaluated using 10,000 bootstrapping method. The area under the receiver operating characteristic (ROC) curve (AUC), calibration curve and decision curve were used to assess the discrimination ability, calibration and clinical benefit of the model, respectively.

\section{Statistical analyses}

Radiomic features were extracted from pelvic CECT using the 3D slicer software. Statistical analyses were conducted by $\mathrm{R}$ software (V 3.6.2). All tests were twosided. A $p$-value $<0.05$ was defined as significant for all the tests except that in multivariate logistic regression with backward elimination strategy a $p$-value $<0.1$ was considered as significant so that potential predictors were less likely to be eliminated from the predictive model.

\section{Results \\ Baseline characteristics}

In total, 75 eligible patients were included in this study according to our inclusion criteria (Fig. 1A). The median $\mathrm{TMB}$ of all patients was $6.5 \mathrm{mut} / \mathrm{Mb}$ (interquartile range: 3.3-12.1). All patients were divided by the median TMB into the high TMB (49.3\%, 37/75) and low TMB (50.7\%, $38 / 75)$ group. The baseline factors were comparable between those with high and low TMB, except that all the seven black patients in this study harbored low TMB (Table 1). The median follow-up time calculated by the reverse Kaplan-Meier method was 29.5 Months. Totally, 
Table 1 Baseline factors of the total cohort and groups with high and low TMB

\begin{tabular}{|c|c|c|c|c|}
\hline & $\begin{array}{l}\text { Total } \\
N=75\end{array}$ & $\begin{array}{l}\text { Low TMB } \\
(N=38)\end{array}$ & $\begin{array}{l}\text { High TMB } \\
(N=37)\end{array}$ & $P$ value \\
\hline \multicolumn{5}{|l|}{ Age $(Y)$} \\
\hline$<69$ & 38 (50.7\%) & $20(52.6 \%)$ & $18(48.6 \%)$ & \multirow[t]{2}{*}{0.730} \\
\hline$\geq 69$ & $37(49.3 \%)$ & 18 (47.4\%) & 19 (51.4\%) & \\
\hline \multicolumn{5}{|l|}{ Gender } \\
\hline Male & 57 (76.0\%) & $28(73.7 \%)$ & 29 (78.4\%) & \multirow[t]{2}{*}{0.634} \\
\hline Female & $18(24.0 \%)$ & 10 (26.3\%) & $8(21.6 \%)$ & \\
\hline \multicolumn{5}{|l|}{ Race } \\
\hline White & 67 (89.3\%) & 31 (81.6\%) & 36 (97.3\%) & \multirow[t]{3}{*}{0.015} \\
\hline Black & 7 (9.3\%) & 7 (18.4\%) & $0(0.0 \%)$ & \\
\hline Asian & $1(1.3 \%)$ & $0(0.0 \%)$ & $1(2.7 \%)$ & \\
\hline \multicolumn{5}{|l|}{ Diagnosis Year } \\
\hline 2005-2010 & $30(40.0 \%)$ & $16(42.1 \%)$ & $14(37.8 \%)$ & \multirow[t]{2}{*}{0.706} \\
\hline 2011-2013 & $45(60.0 \%)$ & 22 (57.9\%) & $23(62.2 \%)$ & \\
\hline \multicolumn{5}{|l|}{ BMI (kg/m2) } \\
\hline$<26.6$ & $34(45.3 \%)$ & 17 (44.7\%) & 17 (45.9\%) & \multirow[t]{3}{*}{0.906} \\
\hline$\geq 26.6$ & $35(46.7 \%)$ & $18(47.4 \%)$ & 17 (45.9\%) & \\
\hline Unknown & $6(8.0 \%)$ & $3(7.9 \%)$ & $3(8.1 \%)$ & \\
\hline \multicolumn{5}{|l|}{ p T stage } \\
\hline pT2 & $24(32.0 \%)$ & $11(28.9 \%)$ & $13(35.1 \%)$ & \multirow[t]{3}{*}{0.676} \\
\hline pT3-4 & $43(57.3 \%)$ & $22(57.9 \%)$ & $21(56.8 \%)$ & \\
\hline Unknown & $8(10.7 \%)$ & $5(13.2 \%)$ & $3(8.1 \%)$ & \\
\hline \multicolumn{5}{|l|}{ p N stage } \\
\hline pNO & $42(56.0 \%)$ & 19 (50.0\%) & $23(62.2 \%)$ & \multirow[t]{3}{*}{0.373} \\
\hline pN1-2 & $21(28.0 \%)$ & 12 (31.6\%) & 9 (24.3\%) & \\
\hline Unknown & $12(16.0 \%)$ & 7 (18.4\%) & $5(13.5 \%)$ & \\
\hline \multicolumn{5}{|l|}{ Stage } \\
\hline Stage II & $28(37.3 \%)$ & $12(31.6 \%)$ & $16(43.2 \%)$ & \multirow[t]{2}{*}{0.296} \\
\hline Stage III & 47 (62.7\%) & $26(68.4 \%)$ & $21(56.8 \%)$ & \\
\hline \multicolumn{5}{|c|}{ Clustering Group } \\
\hline Group A & $24(32.0 \%)$ & $8(21.1 \%)$ & $16(43.2 \%)$ & \multirow[t]{2}{*}{0.039} \\
\hline Group B & $51(68.0 \%)$ & 30 (78.9\%) & $21(56.8 \%)$ & \\
\hline
\end{tabular}

TMB Tumor mutation burden; BMI Body mass index

death and disease progression occurred in 45.3\% (34/75) and $60.0 \%(45 / 75)$ patients, respectively. The median OS and DFS was 35.4 months (95\%CI: 21.6-49.1 months) and 25.0 months (95\%CI: $12.6-37.3$ months), respectively. Log-rank test showed a trend of shorter median OS (35.0 months vs. 56.4 months, $p=0.193$ ) in the low TMB group, although the $p$-value were not significant (Fig. S1 A, B).

\section{Unsupervised hierarchical clustering analysis}

The detailed analysis processes of this study are shown in Fig. 1B. To explore the association between radiomic features and clinical outcomes, we performed an unsupervised hierarchical clustering analysis using the 1223 radiomic features with ICC $>0.8$. As is shown in Fig. 2, the unsupervised clustering divided the whole cohort into two clustering groups (clustering group A: 24/75 [32.0\%]; and clustering group B: 51/75 [68.0\%]) with high radiomic similarity within each group while distinct radiomic profile between groups. The clinical factors were compared between the two groups (Table S1). Of note, compared to cases in the clustering group $\mathrm{B}$, those in group A had a significantly higher proportion of having high TMB (66.7\% [16/24] vs. $41.2 \%$ [21/51], $p=$ 0.039) (Table S1). In addition, when taking TMB as a continuous variable, the median TMB was also higher in the clustering group A against group B (8.31 mut/Mb vs. $4.95 \mathrm{mut} / \mathrm{Mb}, p=0.029$, Fig. 3A). Based on the unsupervised nature of the clustering analysis, these findings indicated that radiomic features extracted from CECT have an intrinsic ability in discriminating the TMB status of BLCA patients. Besides, lower T stage and clinical stage were more frequently found in clustering group A than group B (Table S1). In terms of survival outcomes, patients in clustering group A had more favorable OS against those in group B with a borderline $p$ value (median OS: not reached vs. 33.0, $P=0.058$ Fig. S1. C), while the DFS was also numerically longer in group A (35.7 vs. 19.8, $P=0.257$ Fig. S1. D).

We also explored the relationship between the radiomic features or TMB status with the occurrence of the driver gene mutation. Somatic mutations in genes including TP53, PIK3CA, EP300 and FGFR3 were more frequent in the clustering group A against group B (Fig. $3 \mathrm{~B})$. Besides, high TMB was accompanied by more frequent mutations in TP53, PIK3CA, KMT2C, EP300, ERBB2, ELF3, ERCC2 and BRCA2 gene (Fig. 3C).

\section{Feature selection, model establishment and evaluation}

Since clustering analysis showed the strong potential of radiomic features in predicting $\mathrm{TMB}$, we further tested each feature's power in predicting TMB and developed a TMB-predicting model based on radiomic features. Three sequential steps were involved in the development of the predictive model (Fig. 1B). Firstly, univariate logistic regression analysis was carried out in each radiomic feature. A total of 27 radiomic features with the ability in predicting TMB $(p<0.05)$ were preliminarily identified (Fig. 3D). Then, LASSO regression was conducted using these 27 features to further screen the most powerful prognostic features. 12 radiomic features remained after the LASSO regression (Fig. 3E, F). Afterward, in order to further eliminate the redundant features, these 12 TMB-predicting features were subjected to a backward stepwise logistic regression. Eventually, 6 robust radiomic features were found to be independent 


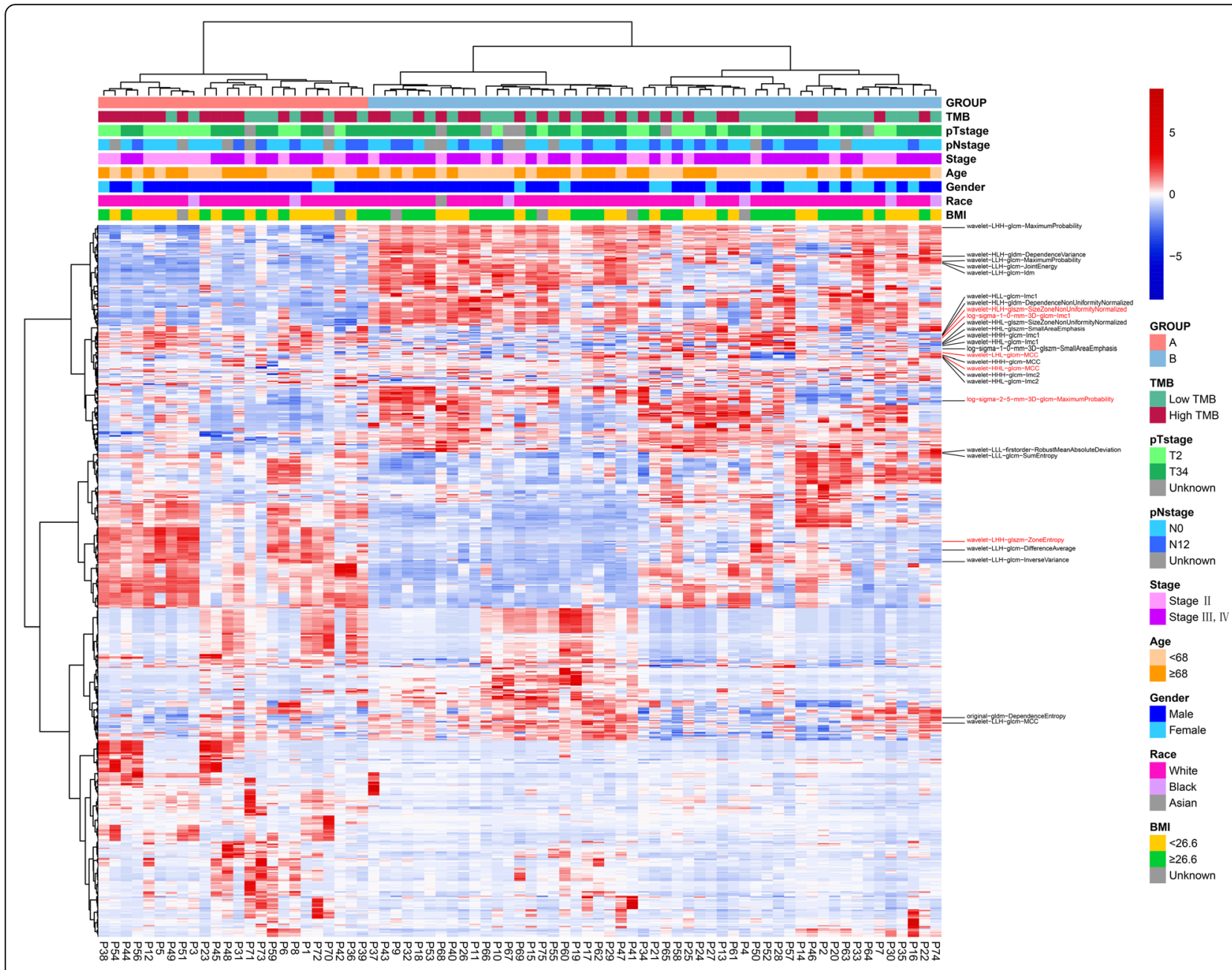

Fig. 2 Unsupervised hierarchical clustering analysis of radiomic features. Radiomic features with predictive ability in the univariate analysis are labeled. Radiomic features included in the final TMB-predicting model are marked in red. Based on the homogeneity and heterogeneity of the radiomic features, all cases were divided into two clustering groups by the unsupervised hierarchical clustering analysis. Clinical and genomic outcomes were compared between the two groups. TMB: Tumor mutation burden; BMI: body mass index

predictors of $\mathrm{TMB}$, of which three and three features were positively and negatively related to high TMB status, respectively (Table 2). Finally, the TMBpredicting model was build based on the 6 radiomic features selected by the backward stepwise regression. Based on the beta value of features included in the backward stepwise regression, a TMB-predicting model of radiomic features was established and visualized as a nomogram (Fig. 4A).

Correlations within distinct radiomic features were also explored. As is shown in Fig. S2A, correlations were identified among several radiomic features with predictive ability in univariate analysis. LASSO regression is widely used to eliminate multi-collinearity. After feature selection by LASSO regression and backward elimination regression, correlations within radiomic features were dramatically reduced (Fig. S2B, C).
The predictive performance of the TMB-predicting model was validated using 10,000 bootstrapping replications in three distinct aspects as follows. The AUC of the ROC curve was 0.853 (95\%CI: 0.770-0.936), reflecting the satisfactory discriminating ability of the model (Fig. 4B). The importance of each radiomic feature in the TMB-predicting model is shown by AUC of ROC curve (Table 2). The highest AUC of ROC curve for a single feature is 0.696. Obviously, the TMB-predicting model harbored much higher predictive accuracy against any radiomic feature alone. Besides, the calibration curve also exhibited good agreement between prediction and observation probability of high TMB (Fig. 4C). In addition, the decision curve analysis demonstrated great positive net benefits among most of the threshold probabilities, indicating the favorable clinical effectiveness of this TMB-predicting model (Fig. 4D). 


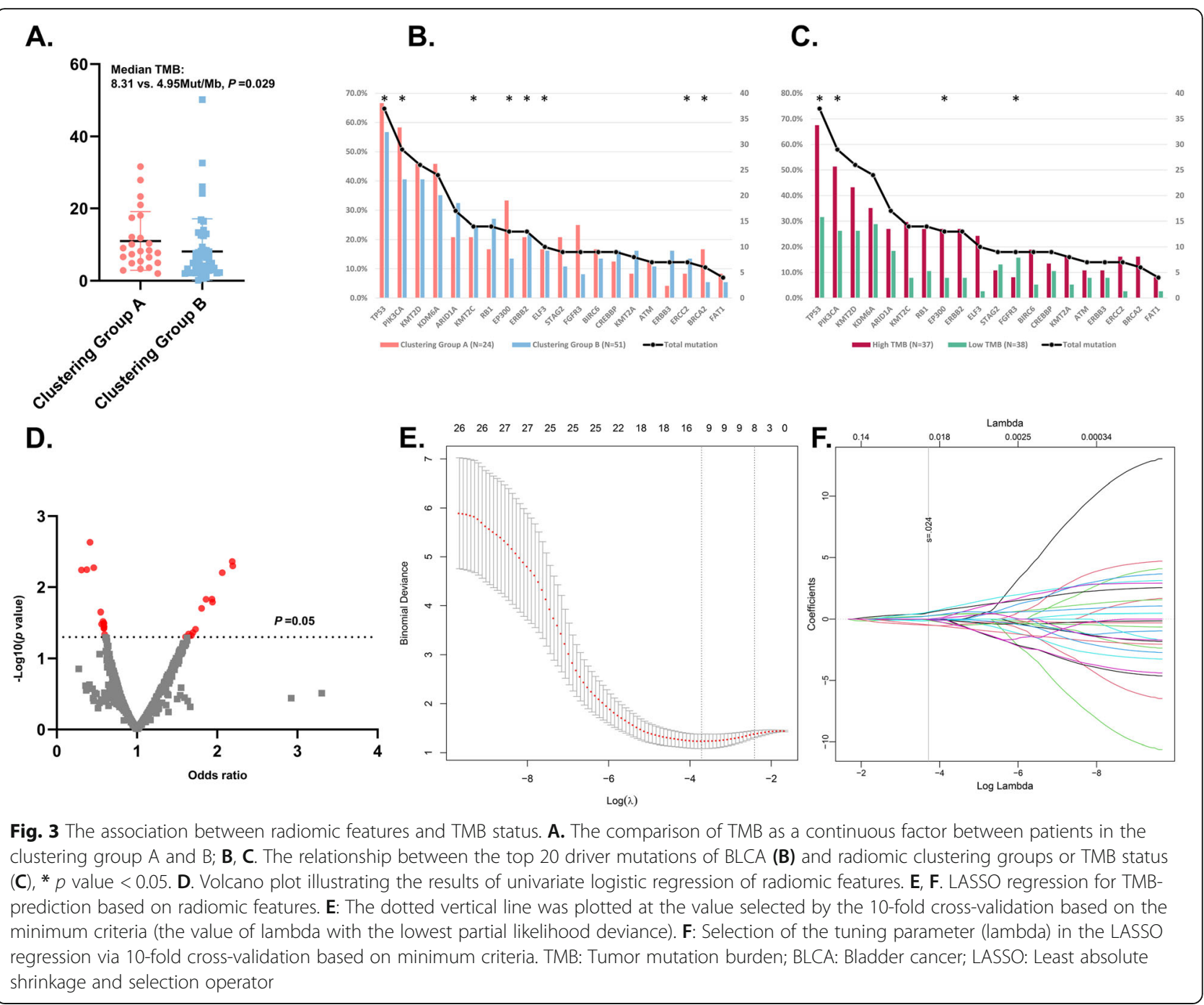

\section{Discussion}

TMB is a widely used therapeutic biomarker for ICIs treatment in many cancers including BLCA [1]. To the best of our knowledge, this study is the first one to investigate the ability of radiomic features extracted from pelvic CECT images to predict the genomic outcomes of BLCA patients. Our findings revealed an intrinsic connection between radiomic features and TMB status as well as several critical driver mutations. In addition, we initiatively developed a radiomic TMB-predicting model that can be used to predict the TMB status of BLCA patients. Though future validation is still needed, our study reveals the practicability of assessing the TMB status by radiomic features for patients with BLCA.

Table 2 Multivariate Logistic regression using backward elimination strategy

\begin{tabular}{|c|c|c|c|c|c|c|c|}
\hline \multicolumn{3}{|l|}{ Radiomic Features } & \multirow{2}{*}{$\begin{array}{l}\text { Beta value } \\
-1.24\end{array}$} & \multirow{2}{*}{$\frac{\text { OR }}{0.29}$} & \multirow{2}{*}{$\begin{array}{l}\mathbf{9 5 \% C l} \text { OR } \\
0.10-0.82\end{array}$} & \multirow{2}{*}{$\begin{array}{l}P \text { value } \\
0.019\end{array}$} & \multirow{2}{*}{$\begin{array}{c}\text { AUC }^{2} \\
0.637\end{array}$} \\
\hline log-sigma-1-0-mm-3D & $\mathrm{glcm}$ & Imc1 & & & & & \\
\hline log-sigma-2-5-mm-3D & $\mathrm{glcm}$ & MaximumProbability & 1.14 & 3.13 & $1.34-7.33$ & 0.009 & 0.638 \\
\hline wavelet-LHL & $\mathrm{glcm}$ & MCC & -1.02 & 0.36 & $0.16-0.82$ & 0.015 & 0.690 \\
\hline wavelet-LHH & glszm & ZoneEntropy & 1.56 & 4.74 & $1.70-13.20$ & 0.003 & 0.661 \\
\hline wavelet-HLH & glszm & SizeZoneNonUniformityNormalized & 1.23 & 3.43 & $1.45-8.13$ & 0.005 & 0.696 \\
\hline wavelet-HHL & $\mathrm{glcm}$ & MCC & -0.80 & 0.45 & $0.18-1.12$ & 0.086 & 0.644 \\
\hline
\end{tabular}

$O R$ Odds ratio, $\mathrm{Cl}$ confidence interval

${ }^{a}$ Area under the receiver operator characteristic curve 


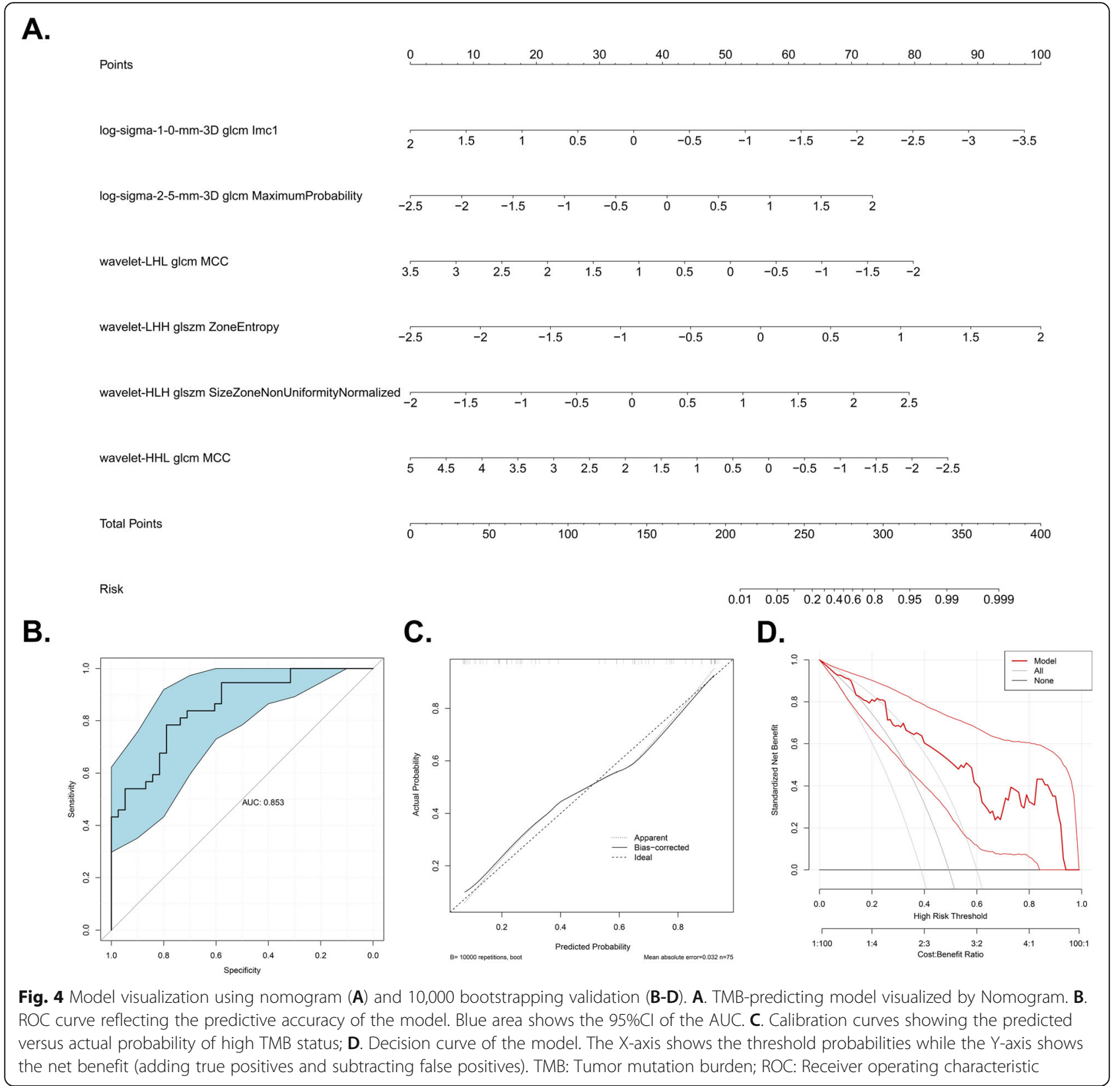

Radiomics study has developed rapidly in a wide range of fields in oncologic researches for its advantages in capturing comprehensive image information [14]. In BLCA, radiomics exhibited promising potential in predicting pathological grade [27], clinical stage [28], lymph node metastasis [29], recurrence [30], progression-free interval [31] and etc. Here, we firstly found that correlations also existed between radiomic features and genomic alterations. Similar findings were observed in patients with lung cancer $[17,19]$. These results implied the possibility that the patient's genetic changes could be reflected on radiological images and quantified by radiomic features.
In our study, the TMB-predicting model was visualized by nomogram which could conveniently calculate the possibility of high TMB for BLCA patients. This tool has the potential in facilitating clinicians to choose the optimal candidates for TMB testing, i.e., patients that are more likely to harbor high TMB. On the other hand, our model could also serve as an alternative of TMB for patients who cannot afford to the expensive TMB testing or does not have accessible tissue sample for the test. Compared to the traditional TMB detection system, our radiomic TMB-predicting model is totally non-invasive. Furthermore, since most BLCA patients have already undergone the pelvic CECT scan before or at initial 
diagnosis, this TMB-predicting model requires almost no additional examination for TMB-testing.

BLCA is a highly immunogenic tumor type due to its high mutational load, and consequently, a promising candidate for immunotherapy. At present, a total of 5 ICIs has been approved to be used as either first-line (atezolizumab and pembrolizumab) or second-line (atezolizumab, durvalumab, avelumab, nivolumab and pembrolizumab) treatment schemes for locally advanced or metastatic BLCA [3]. Given the high cost and nonnegligible toxicity of ICIs, identifying biomarkers that can precisely determine the treatment outcomes of ICIs is of great necessity and importance. As one of the targeting molecules of ICIs, PD-L1 expression is recognized as an ideal marker for therapeutic efficacy prediction [6]. Yet, there are also studies addressing that ICIs can be effective in tumors lacking PD-L1 expression [3, 12]. Apart from $\mathrm{PD}-\mathrm{L} 1, \mathrm{TMB}$ is another promising biomarker. It is well known that the primary targets of human antitumor immune responses are tumor-specific neoantigen peptides originated from somatic mutations in tumors [5]. Accordingly, TMB, which represents the total count of nonsynonymous somatic mutations across the tumor genome, can reflect the antigenicity of tumors. According to a recent meta-analysis, the positive correlations between TMB and ORR in ICIs treatment were found in 27 tumor types [32].

Despite the promising predictive value of TMB in various cancers, TMB detection is now faced with several challenges. One major obstacle for universal TMB testing before ICIs treatment is the high economic cost of the WES examination, which is the golden standard approach for TMB quantifying. Though several relatively cheaper panel-based testing methods have been developed [33, 34], the critical validation of these tools is still lacking. Another annoying aspect of regular WES tests is that it requires an accessible tissue sample which can be hard or even impossible to obtain in some cases. The TMBpredicting model that we established in the current study just makes up for the shortcomings of the above-mentioned traditional TMB detection methods.

This study has several limitations. Firstly, this is a retrospective study with a relatively small sample size, therefore shortcomings connected to its retrospective nature are inevitable. Secondly, since there's currently no consensus about the optimal cut-off value of TMB in BLCA, we chose to use the median TMB to define the high and low TMB status, which could possibly be varied across different centers. Thirdly, the biological explanation behind radiomics' ability to predict TMB in patients with BLCA is not yet known. Future study is still needed to clarify this issue. Finally, although a bootstrapping method was used for the model testing, external validation of the model using data from other centers is still needed.

\section{Conclusion}

In this study, we firstly explored the association between radiomic features and TMB status in patients with BLCA. The results revealed an intrinsic connection between radiomic features extracted from pelvic CECT and TMB status. Besides, we established a radiomic features-based model for TMB-prediction. Our work proved the feasibility of using radiomics to predict TMB. Yet, future study with a larger cohort is needed to verify our findings.

\begin{abstract}
Abbreviations
ICls: Immune checkpoint inhibitors; PD-1: Programmed death-1; BLCA: Bladder cancer; PD-L1: Programmed death-L1; TMB: Tumor mutation burden; WES: whole-exome sequencing; CECT: Contrast-enhanced computed tomography; TCIA: The cancer imaging archive database; TCGA: The cancer genome atlas; VOI: Volume of interest; GLCM: Gray-level co-occurrence matrix; GLDM: Gray-level difference matrix; GLRLM: Gray-level run length matrix; GLSZM: Gray-level size zone; NGTDM: Neighborhood gray tone difference matrix; ICC: Interclass correlation coefficient; LASSO: Least absolute shrinkage and selection operator; ROC: Receiver operating characteristic; AUC: Area under the receiver operating characteristic curve
\end{abstract}

\section{Supplementary Information}

The online version contains supplementary material available at https://doi. org/10.1186/s12885-021-08569-y.

Additional file 1: Table S1. Baseline factors between those of

clustering group $A$ and $B$.

Additional file 2: Fig. S1. Kaplan-Meier curves showing the survival outcomes of all cases. A. Overall survival between patients with high and Iow TMB; B. Disease-free survival between patients with high and low $\mathrm{TMB} ; \mathbf{C}$. Overall survival between patients of clustering group A and B; $\mathbf{D}$. Disease-free survival between patients of clustering group A and B. TMB: Tumor mutation burden. Fig. S2. Correlation analysis among radiomic features sequentially selected by univariate Logistic regression $(\mathbf{A})$, LASSO regression $(\mathbf{B})$ and backward elimination regression $(\mathbf{C})$. Each value represents the correlation coefficient between two radiomic features.

\section{Acknowledgements}

We acknowledge the TCIA and TCGA databases for allowing us to use their freely accessible database.

\section{Authors' contributions}

$X T$ and $Y G$ conceived and designed the study. XT, WQ, WY and TP collected and analyzed the data. XT wrote the manuscript. YG and $Z Y$ revised and edited the manuscript. All authors read and approved the final manuscript.

\section{Funding}

Not applicable.

\section{Availability of data and materials}

The data analyzed in this study are openly available and can be found here: http://www.cancerimagingarchive.net/ (TCIA database); http://cancergenome. nih.gov (TCGA database).

\section{Declarations}

Ethics approval and consent to participate

No ethical approval nor informed consent was required for the current study due to the public availability of data in the TCIA and TCGA databases. 


\section{Consent for publication}

Not applicable.

\section{Competing interests}

The authors declare that they have no competing interests.

Received: 31 March 2021 Accepted: 7 July 2021

Published online: 16 July 2021

\section{References}

1. Havel JJ, Chowell D, Chan TA. The evolving landscape of biomarkers for checkpoint inhibitor immunotherapy. Nat Rev Cancer. 2019;19(3):133-50. https://doi.org/10.1038/s41568-019-0116-x.

2. Aggen $\mathrm{DH}$, Drake CG. Biomarkers for immunotherapy in bladder cancer: a moving target. J Immunother Cancer. 2017;5(1):94. https://doi.org/10.1186/ s40425-017-0299-1.

3. Flaig TW, Spiess PE, Agarwal N, Bangs R, Boorjian SA, Buyyounouski MK, et al. Bladder Cancer, version 3.2020. NCCN Clin Pract Guidel Oncol. 2020; 18(3):329-54.

4. Kennedy LB, Salama AKS. A review of cancer immunotherapy toxicity. CA Cancer J Clin. 2020;70(2):86-104. https://doi.org/10.3322/caac.21596.

5. Fumet JD, Truntzer C, Yarchoan M, Ghiringhelli F. Tumour mutational burden as a biomarker for immunotherapy: current data and emerging concepts. Eur J Cancer. 2020;131:40-50. https://doi.org/10.1016/j.ejca.2020. 02.038 .

6. Patel SP, Kurzrock R. PD-L1 expression as a predictive biomarker in Cancer immunotherapy. Mol Cancer Ther. 2015;14(4):847-56. https://doi.org/10.11 58/1535-7163.Mct-14-0983.

7. Rosenberg JE, Hoffman-Censits J, Powles T, van der Heijden MS, Balar AV, Necchi A, et al. Atezolizumab in patients with locally advanced and metastatic urothelial carcinoma who have progressed following treatment with platinum-based chemotherapy: a single-arm, multicentre, phase 2 trial. Lancet. 2016;387(10031):1909-20. https://doi.org/10.1016/s0140-6736(1 6)00561-4.

8. Samstein RM, Lee $\mathrm{CH}$, Shoushtari AN, Hellmann MD, Shen R, Janjigian YY, et al. Tumor mutational load predicts survival after immunotherapy across multiple cancer types. Nat Genet. 2019;51(2):202-6. https://doi.org/10.1038/ s41588-018-0312-8

9. Rizvi H, Sanchez-Vega F, La K, Chatila W, Jonsson P, Halpenny D, et al. Molecular determinants of response to anti-programmed cell death (PD)-1 and anti-programmed death-ligand 1 (PD-L1) blockade in patients with non-small-cell lung Cancer profiled with targeted next-generation sequencing. J Clin Oncol. 2018;36(7):633-41. https://doi.org/10.1200/jco.201 7.75.3384.

10. Hellmann MD, Callahan MK, Awad MM, Calvo E, Ascierto PA, Atmaca A, et al. Tumor mutational burden and efficacy of Nivolumab Monotherapy and in combination with Ipilimumab in small-cell lung Cancer. Cancer Cell. 2019;35(2):329. https://doi.org/10.1016/j.ccell.2019.01.011.

11. Snyder A, Makarov V, Merghoub T, Yuan J, Zaretsky JM, Desrichard A, et al. Genetic basis for clinical response to CTLA-4 blockade in melanoma. N Engl J Med. 2014;371(23):2189-99. https://doi.org/10.1056/NEJMoa1406498.

12. Balar AV, Galsky MD, Rosenberg JE, Powles T, Petrylak DP, Bellmunt J, et al. Atezolizumab as first-line treatment in cisplatin-ineligible patients with locally advanced and metastatic urothelial carcinoma: a single-arm, multicentre, phase 2 trial. Lancet. 2017;389(10064):67-76. https://doi.org/10.1 016/s0140-6736(16)32455-2.

13. Galsky M, Saci A, Szabo P, Azrilevich A, Horak C, Lambert A, et al. Impact of zumor mutation burden on nivolumab efficacy in second-line urothelial carcinoma patients: exploratory analysis of the phase ii checkmate 275 study. Ann Oncol. 2017;28:v296-v7. https://doi.org/10.1093/annonc/mdx371.003.

14. Avanzo M, Stancanello J, El Naqa I. Beyond imaging: the promise of radiomics. Phys Med. 2017;38:122-39. https://doi.org/10.1016/j.ejmp.2017.05.071.

15. Yang L, Dong D, Fang M, Zhu Y, Zang Y, Liu Z, et al. Can CT-based radiomics signature predict KRAS/NRAS/BRAF mutations in colorectal cancer? Eur Radiol. 2018;28(5):2058-67. https://doi.org/10.1007/s00330-017-5146-8.

16. Li ZC, Bai H, Sun Q, Zhao Y, Lv Y, Zhou J, et al. Multiregional radiomics profiling from multiparametric MRI: identifying an imaging predictor of IDH1 mutation status in glioblastoma. Cancer Med. 2018;7(12):5999-6009. https://doi.org/10.1002/cam4.1863.

17. Tu W, Sun G, Fan L, Wang Y, Xia Y, Guan Y, et al. Radiomics signature: a potential and incremental predictor for EGFR mutation status in NSCLC patients, comparison with CT morphology. Lung Cancer. 2019;132:28-35. https://doi.org/10.1016/j.lungcan.2019.03.025.

18. Zhang N, Wu J, Yu J, Zhu H, Yang M, Li R. Integrating imaging, histologic, and genetic features to predict tumor mutation burden of non-small-cell lung Cancer. Clin Lung Cancer. 2020;21(3):e151-e63. https://doi.org/10.1016/ j.cllc.2019.10.016.

19. Wang X, Kong C, Xu W, Yang S, Shi D, Zhang J, et al. Decoding tumor mutation burden and driver mutations in early stage lung adenocarcinoma using CT-based radiomics signature. Thorac Cancer. 2019;10(10):1904-12. https://doi.org/10.1111/1759-7714.13163.

20. Clark K, Vendt B, Smith K, Freymann J, Kirby J, Koppel P, et al. The Cancer imaging archive (TCIA): maintaining and operating a public information repository. J Digit Imaging. 2013;26(6):1045-57. https://doi.org/10.1007/s102 78-013-9622-7.

21. Cancer Genome Atlas Research Network, Weinstein JN, Collisson EA, Mills GB, Shaw KR, Ozenberger BA, et al. The Cancer Genome Atlas Pan-Cancer analysis project. Nat Genet. 2013;45(10):1113-20. https://doi.org/10.1038/ng.2764.

22. Fedorov A, Beichel R, Kalpathy-Cramer J, Finet J, Fillion-Robin JC, Pujol S, et al. 3D slicer as an image computing platform for the quantitative imaging network. Magn Reson Imaging. 2012;30(9):1323-41. https://doi. org/10.1016/j.mri.2012.05.001.

23. Mahon R, Ghita M, Hugo G, Weiss E. ComBat harmonization for radiomic features in independent phantom and lung cancer patient computed tomography datasets. Phys Med Biol. 2020;65(1):015010. https://doi.org/10.1 088/1361-6560/ab6177.

24. Orlhac F, Boughdad S, Philippe C, Stalla-Bourdillon H, Nioche C, Champion $L$, et al. A Postreconstruction harmonization method for multicenter Radiomic studies in PET. J Nucl Med. 2018;59(8):1321-8. https://doi.org/10.2 967/jnumed.117.199935

25. Chalmers ZR, Connelly CF, Fabrizio D, Gay L, Ali SM, Ennis R, et al. Analysis of 100,000 human cancer genomes reveals the landscape of tumor mutational burden. Genome Med. 2017;9(1):34. https://doi.org/10.1186/s13 073-017-0424-2.

26. Gonzalez-Perez A, Perez-Llamas C, Deu-Pons J, Tamborero D, Schroeder MP, Jene-Sanz A, et al. IntOGen-mutations identifies cancer drivers across tumor types. Nat Methods. 2013;10(11):1081-2. https://doi.org/10.1038/nmeth.2642.

27. Wang H, Hu D, Yao H, Chen M, Li S, Chen H, et al. Radiomics analysis of multiparametric MRI for the preoperative evaluation of pathological grade in bladder cancer tumors. Eur Radiol. 2019;29(11):6182-90. https://doi.org/1 0.1007/s00330-019-06222-8.

28. Lim CS, Tirumani S, van der Pol CB, Alessandrino F, Sonpavde GP, Silverman SG, et al. Use of quantitative T2-weighted and apparent diffusion coefficient texture features of bladder Cancer and Extravesical fat for local tumor staging after transurethral resection. AJR Am J Roentgenol. 2019;212(5):1-10. https://doi.org/10.2214/ajr.18.20718

29. Wu S, Zheng J, Li Y, Wu Z, Shi S, Huang M, et al. Development and validation of an MRI-based Radiomics signature for the preoperative prediction of lymph node metastasis in bladder Cancer. EBioMedicine. 2018; 34:76-84. https://doi.org/10.1016/j.ebiom.2018.07.029.

30. Xu X, Wang H, Du P, Zhang F, Li S, Zhang Z, et al. A predictive nomogram for individualized recurrence stratification of bladder cancer using multiparametric MRI and clinical risk factors. J Magn Reson Imaging. 2019; 50(6):1893-904. https://doi.org/10.1002/jmri.26749.

31. Lin P, Wen DY, Chen L, Li X, Li SH, Yan HB, et al. A radiogenomics signature for predicting the clinical outcome of bladder urothelial carcinoma. Eur Radiol. 2020;30(1):547-57. https://doi.org/10.1007/s00330-019-06371-w.

32. Yarchoan M, Hopkins A, Jaffee EM. Tumor mutational burden and response rate to PD-1 inhibition. N Engl J Med. 2017;377(25):2500-1. https://doi.org/1 0.1056/NEJMc1713444

33. Stenzinger A, Endris V, Budczies J, Merkelbach-Bruse S, Kazdal D, Dietmaier W, et al. Harmonization and standardization of panel-based tumor mutational burden measurement: real-world results and recommendations of the quality in pathology study. J Thorac Oncol. 2020;15(7):1177-89. https://doi.org/10.1016/j.jtho.2020.01.023.

34. Zhuang W, Ma J, Chen X, Wang G, Lu J, Chen Y, et al. The tumor mutational burden of Chinese advanced Cancer patients estimated by a 381-cancergene panel. J Cancer. 2018;9(13):2302-7. https://doi.org/10.7150/jca.24932.

\section{Publisher's Note}

Springer Nature remains neutral with regard to jurisdictional claims in published maps and institutional affiliations. 